\title{
Correlation of the ganglionar and metastasic stages, before and after the surgery, of the cases of colon cancer diagnosed at the Universitary hospital of Puerto real, period 2015-17
}

Keywords: colon cancer, breast cancer, industrialized areas, colon tumors, patients

Abbreviations: CC, colon cancer; AJCC, American joint committee on cancer; UICC, international union against cancer

\section{Introduction}

Colon cancer $(\mathrm{CC})$ is the second tumor in incidence after lung cancer in men and only proceeded by breast cancer in women. Mortality and incidence is highest in industrialized areas. The majority of colon tumors are adenocarcinoma that originate from the mucosa and are located mainly in the left and distal colon, although an increase in the incidence of right colon tumors is also being reported. ${ }^{1,2}$ $\mathrm{CC}$ has a high cure rate when it presents as localized disease and radical surgical treatment is practiced. ${ }^{3}$ However, the recurrence of the disease after surgery is the most imperative problem and the main cause of death in most patients. Therefore the prognosis is determined by factors such as: level of invasion of the colon wall, extension to loco regional lymph nodes or by the presence of metastasis in target organs, these three elements represent the basis of the TNM staging system, method of staging of neoplasms developed by the AJCC (American Joint Committee on Cancer) in collaboration with the UICC (International Union Against Cancer). ${ }^{4}$ The goal is the correct staging of the different tumors focused on clinical management, therapeutic decision, evaluation after treatment, prognosis and unification of criteria for research projects and data transmission between centers.

The abdominal computerized axial tomography is essential for the TNM staging system; it assesses the regional extension of the tumor, as well as the presence of lymphadenopathy and metastasis, and also highlites complications of the neoplasia such as obstruction, perforation and fistula. ${ }^{5}$

\section{Objective of the study}

The aim of our study is to analyze the correlation between clinical staging and pathological staging performed after surgery of patients diagnosed with colon adenocarcinoma, specifically assessing the presence of lymphadenopathy and the presence of metastatic lesions, as well as the role of computerized axial tomography (TC), as a method of analyzing pre-surgical stratification in this type of tumor.

\section{Material and methods}

A descriptive, retrospective, epidemiological study of all cases of colon cancer undergoing digestive surgery at the University Hospital of Puerto Real in the period 2015-2017, analysis of 3years. All the patients had a histological diagnosis of adenocarcinoma. The capacity of the CT for the lymph node staging and for the metastatic staging was determined and the results were compared with those obtained in the pathological anatomy after the surgical resection. The data was analyzed in the SPSS computer statistical program (Version 15.0)
Volume 10 Issue I - 2019

\author{
Marta Rivas Rivas, Germán Santamaría \\ Rodríguez,Victoria de Lara Behaman, Coral \\ de la Vega Olias \\ Hospital Universitario de Puerto real (CÁDIZ), Spain
}

\author{
Correspondence: Germán Santamaría Rodríguez, Hospital \\ Universitario de Puerto real (CÁDIZ), Spain, \\ Email germansrv@gmail.com
}

Received: August 01, 2018 | Published: January 17, 2019

\section{Results}

The estimation with the TAC of the abdomen in the presence of adenopathies in colon cancer was correct in $66.4 \%$ (97) of the cases, depending mostly on the diagnosis of the negative nodes, true negative 65 cases, true positives 32 cases. In $33.6 \%$ of the cases, the lymph node diagnosis was erroneous, under diagnosed in $15.1 \%$ (22) of the cases and overstated in $18.5 \%$ (27) of the cases (Table 1). Regarding the presence of metastasis, the diagnosis was correct in $94.5 \%$ of the cases, depending almost entirely on the absence of metastatic lesions, the diagnosis was underestimated in $2.7 \%$ (4) of the cases and overstated also in $2.7 \%$ of the cases (Table2). The sensitivity of the CT for the presence of adenopathies in colon cancer is $59 \%$ and the specificity $70 \%$ (Table 3 ) and for the presence of metastasis the sensitivity is $73 \%$, the specificity $96 \%$ (Table 4 ).

Table I Comparison of the clinical diagnosis (before the surgery) and the pathological diagnosis ( post- surgery) of the presence of adenopathies in the tumor extension study of the CRC.

\begin{tabular}{lll}
\hline Adenopathies Nc y Np & N & \% Valid \\
\hline Correct Diagnosis & 97 & 66,4 \\
Overdiagnosed & 27 & 18,5 \\
Infradiagnosed & 22 & 15,1 \\
Total & 146 & 100 \\
\hline
\end{tabular}

Result: In $66 \%$ of the cases there has been a correct diagnosis, in $18.5 \%$ we have given as pathological lymphadenopathies the lymphadenopathies that were inflammatory, and in $15.1 \%$ we have not diagnosed pathological lymphadenopathies when they were.

Table 2 Comparison of clinical (before surgery) and pathological diagnosis (after surgery) the presence of metastasis.

\begin{tabular}{lll}
\hline Metastasis & N & \% Valid \\
\hline Correct Diagnosis & 138 & 94,5 \\
Over diagnosed & 4 & 2,7 \\
Infra diagnosed & 4 & 2,7 \\
Total & 146 & 100
\end{tabular}

Result: We have made a correct diagnosis in $94.5 \%$ of the cases. In $4 \%$ of cases we diagnosed a disease as metastatic when it wasn't, and in $4 \%$ of cases it was misdiagnosed the presence of metastases when they really were present. 
Table 3 Ganglionar affection according to SCAN diagnosis.

\begin{tabular}{lll}
\hline Ganglionar affection & Positive cases & Negative cases \\
\hline Positive TC ( Scan) & 32 & 27 \\
Negative TC & 22 & 65 \\
\hline \multicolumn{2}{c}{ Sensibility } & $59 \%$ \\
Specificity & $70 \%$ \\
VPP ( positive predictive value) & $54 \%$ \\
VPN ( negative predictive value) & $74 \%$
\end{tabular}

The probability that a patient with pathological lymphadenopathies will obtain a positive result in the test is $59 \%$. The probability for a subject without pathological lymphadenopathies would have a Negative result in the test is $70 \%$.

In $54 \%$ of patients with pathological adenopathies, adenopathies were finally corrected diagnosed, whilst in $74 \%$ of patients who did not have pathological lymph nodes at Scan images, in fact they were not present.

Table 4 Metastatic affection according to SCAN diagnosis.

\begin{tabular}{|c|c|c|c|}
\hline \multicolumn{2}{|l|}{ Metastatic affection } & Positive cases & \multirow{2}{*}{$\begin{array}{l}\text { Negative cases } \\
4\end{array}$} \\
\hline Positive TC & & 11 & \\
\hline \multirow[t]{5}{*}{ Negative TC } & & 4 & 127 \\
\hline & Sensibility & $73 \%$ & \\
\hline & Specificity & $96 \%$ & \\
\hline & $V P P$ & $73 \%$ & \\
\hline & $V P N$ & $96 \%$ & \\
\hline
\end{tabular}

The probability for a subject with metastasis obtaining a positive result in the test is of $73 \%$. The probability for a patient without metastasis a negative result will be obtained in the test is $96 \%$.

In $73 \%$ of patients with metastases these metastases were finally correctly diagnosed, whilst in $96 \%$ of patients in whom metastases were not identified at the initial clinical diagnosis finally did not have them.

\section{Conclusion}

The probability that a patient with lymph node involvement will obtain a positive result on computerized tomography is $59 \%$ (sensitivity of CT to the presence of lymphadenopathy) and the probability that a patient with metastasis obtains a positive result is $73 \%$ (sensitivity of the CT to the presence of metastasis).
The probability that a patient without lymph node involvement will obtain a negative result is $70 \%$ (specificity of the CT for the diagnosis of adenopathies) and the probability that a patient without metastasis will obtain a negative result is $96 \%$ (specificity of the $\mathrm{CT}$ scan to the diagnosis of metastasis). In summary sensitivity and specificity was greater for the diagnosis of metastasis than for the lymph node diagnosis, the specificity was greater for both cases, that is to be expected in the confirmatory diagnostic tests to avoid serious physical, psychological and economic consequences, the results obtained are similar to the few studies published in the literature. ${ }^{6,7}$

\section{Acknowledgments}

None.

\section{Conflicts of interest}

The author declares no conflicts of interest.

\section{References}

1. Binefa G, Rodríguez-Moranta F, Teule A, et al. Colorectal cancer: from prevention to personalized medicine. World $J$ Gastroenterol. 2014;20(22):6786-6808.

2. Ministerio de Sanidad y Consumo. La situación del cáncer en España. Centro Nacional de Epidemiología. Instituto de Salud Carlos III. Madrid, España; 2005.

3. Schmoll HJ, Van Cutsem E, Stein A, et al. ESMO Consensus Guidelines for management of patients with colon and rectal cancer. A personalized approach to clinical decision making. Annals of Oncology. 2012;23(10):2479-2516.

4. Harry B Burke. Outcome Prediction and the Future of the TNM Staging System. J Natl Cancer Inst. 2004;96(19):1420-1425.

5. Horton KM, Abrams RA, Fishman EK. Spiral CT of colon cancer: imaging in management. Radiographics. 2000;20(22):419-430.

6. Sali L, Falchini M, Taddei A, et al. Role of preoperative CT colonography in patients with colorectal cancer. World J Gastroenterol. 20(14):3795-3803.

7. Filippone A, Ambrosini R, Fuschi M, et al. Preoperative T and N staging of colorectal cancer: accuracy of contrast-enhanced multi-detector row CT colonography_initial experience. Radiology. 2004;231(1):83-90. 